\title{
Introduction of a qualitative perinatal audit at Muhimbili National Hospital, Dar es Salaam, Tanzania
} Hussein L Kidanto*1,2,3, Ingrid Mogren ${ }^{5}$, Jos van Roosmalen ${ }^{4}$, Angela N Thomas ${ }^{2}$, Siriel N Massawe ${ }^{1,2}$, Lennarth Nystrom $^{3}$ and Gunilla Lindmark ${ }^{6}$

\author{
Address: ${ }^{1}$ Department of Obstetrics and Gynaecology, Muhimbili University of Health and Allied Sciences, Dar es Salaam, Tanzania, ${ }^{2}$ Department \\ of Obstetrics and Gynaecology, Muhimbili National Hospital, Dar es Salaam, Tanzania, ${ }^{3}$ Department of Public Health and Clinical Medicine, \\ Epidemiology and Public Health Sciences, Umeå University, Umeå, Sweden, ${ }^{4}$ Department of Obstetrics, Leiden University Medical Centre and \\ Section of Health care and Culture, VU University Medical Centre, Amsterdam, The Netherlands, ${ }^{5}$ Department of Clinical Science, Obstetrics and \\ Gynaecology, Umeå University, Umeå, Sweden and ' ${ }^{2}$ epartment of Women's and Children's Health, Academic Hospital, Uppsala, Sweden \\ Email: Hussein L Kidanto* - hkidanto@yahoo.co.uk; Ingrid Mogren - ingrid.mogren@obgyn.umu.se; Jos van \\ Roosmalen - j.j.m.van_roosmalen@lumc.nl; Angela N Thomas - angluma@yahoo.com; Siriel N Massawe - smassawe@muhas.ac.tz; \\ Lennarth Nystrom - Lennarth.Nystrom@epiph.umu.se; Gunilla Lindmark - Gunilla.Lindmark@kbh.uu.se \\ * Corresponding author
}

Published: 19 September 2009

BMC Pregnancy and Childbirth 2009, 9:45 doi:10.1 186/147|-2393-9-45

This article is available from: http://www.biomedcentral.com//47/-2393/9/45

(c) 2009 Kidanto et al; licensee BioMed Central Ltd.

This is an Open Access article distributed under the terms of the Creative Commons Attribution License (http://creativecommons.org/licenses/by/2.0), which permits unrestricted use, distribution, and reproduction in any medium, provided the original work is properly cited.
Received: 27 May 2009
Accepted: 19 September 2009

\begin{abstract}
Background: Perinatal death is a devastating experience for the mother and of concern in clinical practice. Regular perinatal audit may identify suboptimal care related to perinatal deaths and thus appropriate measures for its reduction. The aim of this study was to perform a qualitative perinatal audit of intrapartum and early neonatal deaths and propose means of reducing the perinatal mortality rate (PMR).
\end{abstract}

Methods: From |st August, 2007 to $\left.3\right|^{\text {st }}$ December, 2007 we conducted an audit of perinatal deaths $(\mathrm{n}=133)$ with birth weight $1500 \mathrm{~g}$ or more at Muhimbili National Hospital $(\mathrm{MNH})$. The audit was done by three obstetricians, two external and one internal auditors. Each auditor independently evaluated the cases narratives. Suboptimal factors were identified in the antepartum, intrapartum and early neonatal period and classified into three levels of delay (community, infrastructure and health care). The contribution of each suboptimal factor to adverse perinatal outcome was identified and the case graded according to possible avoidability. Degree of agreement between auditors was assessed by the kappa coefficient.

Results: The PMR was 92 per 1000 total births. Suboptimal factors were identified in $80 \%$ of audited cases and half of suboptimal factors were found to be the likely cause of adverse perinatal outcome and were preventable. Poor foetal heart monitoring during labour was indirectly associated with over $40 \%$ of perinatal death. There was a poor to fair agreement between external and internal auditors.

Conclusion: There are significant areas of care that need improvement. Poor monitoring during labour was a major cause of avoidable perinatal mortality. This type of audit was a good starting point for quality assurance at $\mathrm{MNH}$. Regular perinatal audits to identify avoidable causes of perinatal deaths with feed back to the staff may be a useful strategy to reduce perinatal mortality. 


\section{Background}

The perinatal mortality rate (PMR) in Tanzania is among the highest in the world. Therefore, to reduce perinatal mortality needs a major effort in order to achieve the Millennium Development Goal no. 4 to reduce child mortality by two third (MDG4). Although the Tanzania Demographic and Health Survey [1] showed a decrease in under - 5- mortality from 147 deaths/1000 in 1994 to 1999 to 112 deaths/1000 in 2000 to 2004 , the neonatal mortality rate (32/1000 live births) had not declined. The reduction of child deaths can only be achieved if perinatal survival is improved; several studies have indicated up to half of the perinatal deaths globally occur as a direct consequence of poorly managed deliveries [2-4].

Hospital-based studies in low income countries have shown that 3 out of 4 perinatal deaths may be due to suboptimal care $[5,6]$. Our previous study in Dar es Salaam 1999-2003 estimated the PMR at 123 per 1000 total births and the majority of these deaths were assumed preventable [6]. Thus, reduction of PMR and improvement of maternal and child health requires identification of service-related factors leading to perinatal deaths $[7,8]$. One approach is to perform clinical audits in obstetric care, i.e. retrospective critical reviews of clinically undesirable pregnancy-related events.

Perinatal mortality audits in obstetrics are intended to determine primary and final causes of death as well as suboptimal factors and missed opportunities to ascertain how to improve future management. Preventable factors could be health professional related, such as a health provider failing to perform recommended procedures, or be administration related, such as unavailability of necessary drugs, other preventable factors could be patient-related, such as delay to seek medical assistance due to various reasons [9]. The fundamental goal of establishing perinatal audits in areas with high PMR is to reduce the number of perinatal deaths through an improvement in the quality of care. Several studies $[10,11]$ have shown a strong association between the establishment of an effective audit process and improvement of the quality of maternal health services and a reduction of maternal and perinatal foetal mortality rates.

The aim of this study was to introduce a qualitative perinatal audit in an urban tertiary centre in Tanzania, the main focus being on obstetric care during labour and delivery.

\section{Methods \\ Setting}

The study was carried out in the labour ward at Muhimbili National Hospital (MNH), a teaching hospital for Muhimbili University College of Health Sciences and one of four large consultant hospitals in the United Republic of Tanzania. It is situated in Dar es Salaam, which has a population of about 2.5 million and an annual population growth rate of $4.3 \%$ [1]. The hospital serves as a referral centre for the city of Dar es Salaam and the neighbouring coastal region. Annual number of deliveries was about 10,000, corresponding to about 30 deliveries per day, out of which $80 \%$ are low-risk deliveries. Every month there is a perinatal mortality meeting involving all members of the department of obstetrics and representatives from the neonatal unit, where the monthly trend as well as a selected perinatal mortality case is discussed, however not in the form of a formalised audit.

There are three shifts for nurses working in the labour ward, each with six midwives. One specialist obstetrician, one consultant obstetrician and one resident (house officer) are on call every day. After a normal uneventful vaginal delivery the mothers and babies are often observed in hospital for 6-10 hours. During this time the babies also get BCG and polio vaccinations before being discharged. Babies delivered by caesarean section (CS) or those with low Apgar score $(<7)$ were admitted to the neonatal ward, which was just one floor up from the labour ward. The unit also admitted sick babies from other hospitals.

\section{Material}

Information on all perinatal deaths occurring from $1^{\text {st }}$ August, 2007 to $30^{\text {th }}$ December 2007 were collected through case notes, antenatal cards, and maternity midwifery registry records and classified according to the modified Nordic Baltic classification [12]. A check list was used to make sure that all the required information was obtained. For each stillbirth and early neonatal death, information was abstracted on the date of birth, residential area of the woman, antenatal care attendance, maternal age, parity, estimated gestational age, birth weight, sex and vital status of the baby at birth (Apgar score at 1 and 5 minutes), multiple births, and mode of delivery. Women with at least one antenatal care (ANC) visit were considered as having received antenatal care.

Perinatal death was defined according to the World Health Organization (WHO) 1997 definition of viability, i.e. a birth weight of $\geq 500$ grams. However, for the purpose of this paper only babies weighing $\geq 1500$ grams were included in the analysis as the neonatal unit of MNH adequately can take care of this group of newborns. The neonates admitted to the neonatal unit soon after the delivery was followed for 7 days in order to record early neonatal deaths.

\section{Audit procedure}

The audit was performed by obstetricians to focus on the care given during labour and delivery from an obstetric perspective. Cases of stillbirth and early neonatal death 
weighing $\geq 1500$ grams were assessed through narratives from case notes by an expert panel of two external and one internal auditor. Narratives from the case notes and grading forms were prepared in English by the first author and dispatched to the internal auditor and the two external auditors in the Netherlands and Sweden. The auditor from the Netherlands had worked in Tanzania before and has vast experience on African health systems whereas the auditor from Sweden never had worked in Africa.

The audit protocol was prepared and agreed at a perinatal audit workshop convened at the hospital. This workshop was attended by members of the department i.e. nurses, midwives and doctors as well as the auditors. The role of the audit panel members was to identify those situations that were critical and required action. If the required action was not covered by the objective criteria that were formulated beforehand, it was up to the personal judgment of the panel member to assess the adequacy of action taken and to comment on the level of sub-optimality. Suboptimal factors were identified in the antepartum, intrapartum and neonatal periods, and classified in three levels of delay:
1. Maternal/social factors (delay related to the patient or relatives).

2. Communication/infrastructure (delay due to transport problem).

3. Health care factors (Delay of appropriate care after admission to hospital).

The auditors worked independently using a structured assessment protocol and grading form. The contribution of each suboptimal factor to the fatal outcome was assessed and a final grade (Table 1) was given by each auditor. After collating the assessment forms the coordinator (corresponding author) computerized the information.

\section{Statistical analysis}

Degree of agreement between auditors was assessed by the kappa coefficient using the shareware Win Pepi. We adopted the Landis and Koch scale [13] i.e. a kappa coefficient of 0-0.20 indicates poor agreement, 0.21-0.40 fair

Table I: Perinatal audit form for identification of suboptimal factors and grading

\begin{tabular}{|c|c|c|}
\hline Item & Choice & Selection \\
\hline \multirow[t]{6}{*}{ Level of delay } & I = Maternal social (community level) & \\
\hline & 2 = Infrastructure service organization & \\
\hline & $3=$ Care & \\
\hline & $4=$ Infrastructure and care & \\
\hline & $5=$ All of above & \\
\hline & $6=$ No delay & \\
\hline \multirow[t]{3}{*}{ Contribution of the suboptimal care to the foetal death } & I = Unlikely & \\
\hline & 2 = Possibly & \\
\hline & 3 = Likely & \\
\hline \multirow[t]{4}{*}{ Final grading } & Grade 0 -No suboptimal care identified & \\
\hline & $\begin{array}{l}\text { Grade I -Suboptimal care, identified but } \\
\text { unlikely to have contributed to the fatal } \\
\text { outcome, different management would } \\
\text { have made no difference to the outcome }\end{array}$ & \\
\hline & $\begin{array}{l}\text { Grade II -Suboptimal care identified and } \\
\text { might have contributed to the fatal } \\
\text { outcome. Different management might } \\
\text { have made a difference to the outcome. }\end{array}$ & \\
\hline & $\begin{array}{l}\text { Grade III -Suboptimal care identified } \\
\text { and is likely to have contributed to the } \\
\text { fatal outcome. Different management } \\
\text { would reasonably be expected to have } \\
\text { made a difference to the outcome. A } \\
\text { clearly avoidable factor implying that the } \\
\text { adverse outcome could have been } \\
\text { prevented. }\end{array}$ & \\
\hline \multicolumn{3}{|l|}{ Overall suboptimal graded $0, \mathrm{I}, \mathrm{II}$ or III according to above definition } \\
\hline Do you think there was sufficient information available to assign a final grade in this case & $\begin{array}{l}1=\text { Yes } \\
2=\text { No }\end{array}$ & \\
\hline Do you consider likely that this death was preventable? & $\begin{array}{l}1=\text { Yes } \\
2=\text { No }\end{array}$ & \\
\hline Number of panel member..................... & 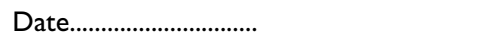 & \\
\hline
\end{tabular}




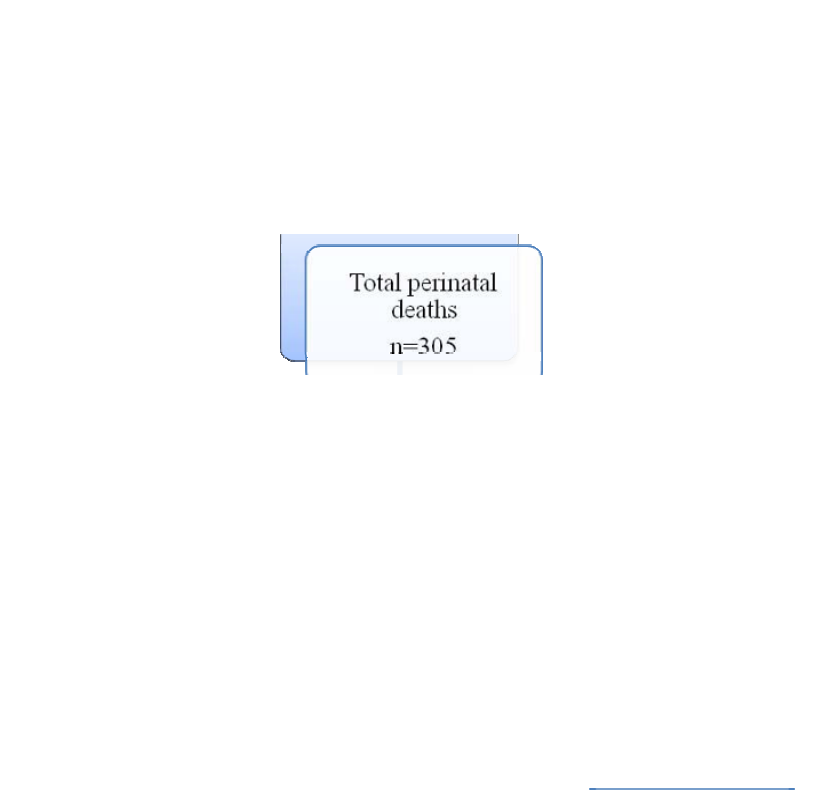

Figure I

Cases sorting flow chart.

agreement, 0.41-0.60 moderate agreement, 0.61-0.80 good agreement, and $0.81-1$ very good agreement.

\section{Results}

During the study period of five months 3767 births were recorded out of which 3449 were live births. There was a total of 240 stillbirth of which 120 cases were macerated pre-labour stillbirth, 120 cases of fresh intrapartum stillbirth and 65 cases of early neonatal death, thirteen cases were unclassified, therefore there were 305 perinatal deaths (Figure 1).

The stillbirth, early neonatal mortality and overall perinatal mortality rates in the material were 64/1000 births, 19/ 1000 live births and 81/1000 births, respectively. Perinatal deaths were significantly more common among teenage mothers (33\% vs. $13 \%$; $\mathrm{p}<0.001$ ), and in preterm babies (54\% vs. $18 \%$; p < 0.0001; Table 2). However, almost half of the deaths were babies born at term.

For the audit purpose out of 305 perinatal deaths we excluded 111 babies weighing <1500 grams (73 macerated stillbirths, 20 fresh stillbirths, 18 neonatal deaths) as well as all unclassified cases. Finally, we excluded an additional 61 perinatal deaths with missing values or case note (29 macerated stillbirths, 22 fresh stillbirths, 10 neonatal deaths) hence leaving 133 cases for the audit (corresponding to $68.5 \%$ of perinatal deaths weighing $1.5 \mathrm{~kg}$ or $43.6 \%$ of total perinatal deaths during the study period); Figure 1).
Table 2: Maternal characteristics of women who experienced perinatal death at Muhimbili National Hospital, AugustDecember 2007

\begin{tabular}{|c|c|c|c|c|}
\hline \multirow[t]{2}{*}{ Maternal characteristic } & \multicolumn{2}{|c|}{ Perinatal deaths } & \multicolumn{2}{|c|}{ All births } \\
\hline & $\mathbf{n}$ & $\%$ & $\mathbf{n}$ & $\%$ \\
\hline \multicolumn{5}{|l|}{ Age (years) } \\
\hline $13-19$ & 100 & 32.8 & 487 & 13.0 \\
\hline $20-29$ & 61 & 20.0 & 1086 & 28.9 \\
\hline $30-39$ & 132 & 43.3 & 2055 & 54.7 \\
\hline$\geq 40$ & 12 & 3.9 & 126 & 3.4 \\
\hline \multicolumn{5}{|l|}{ Parity } \\
\hline 0 & 121 & 39.7 & 1507 & 40.2 \\
\hline $\mathrm{I}-2$ & 123 & 40.3 & 1689 & 45.0 \\
\hline$\geq 3$ & 61 & 20.0 & 558 & 14.8 \\
\hline \multicolumn{5}{|l|}{ Gestational age (weeks) } \\
\hline $28-33$ & 93 & 30.5 & 168 & 4.5 \\
\hline $34-36$ & 70 & 23.0 & 518 & 13.8 \\
\hline$\geq 37$ & 142 & 46.6 & 3068 & 81.7 \\
\hline \multicolumn{5}{|l|}{ No of ANC visits } \\
\hline 0 & 0 & 0.0 & 1 & 0.0 \\
\hline $1-3$ & 155 & 50.8 & 1333 & 35.5 \\
\hline$\geq 4$ & 150 & 49.2 & 2420 & 64.5 \\
\hline \multicolumn{5}{|l|}{ Source of admission } \\
\hline Home & 153 & 50.2 & 762 & 20.3 \\
\hline Hospital transfer & 152 & 49.8 & 2992 & 79.7 \\
\hline Total & 305 & 100 & 3754 & 100 \\
\hline
\end{tabular}

Table 3 summarizes the causes of perinatal deaths and maternal medical conditions. One third of the patients were admitted without audible foetal heart beats. Birth asphyxia was the main cause of intrapartum fresh stillbirth (47\%) and early neonatal death (51\%), whereas eclampsia (25\%) anaemia (14\%) and preeclampsia $(8.3 \%)$ were the main maternal medical conditions. The majority of stillbirths were fresh, indicating foetal demise during labour or less than 24 hours before delivery.

All three auditors identified suboptimal factors in about $80 \%$ of audited cases out of which about $50 \%$ were found to be the likely cause of the adverse perinatal outcome (Table 4). The external auditors I and II reported 53\% and $44 \%$ of the deaths as associated to suboptimal care as compared to $44 \%$ for the internal auditor (Table 4 ). Most cases (37\% and $43 \%$ ) were given a final grade III by external auditors I and II as compared to $26 \%$ by the internal auditor. In 22 cases auditors did not find evidence of suboptimal care $(15,19$, and 22 cases for external auditors 2, 1 and internal auditor respectively.

The external auditors agree that over $75 \%$ of audited cases the death was preventable as compared to $52 \%$ by the internal auditor. Furthermore, all auditors considered the information provided as sufficient to assign the final grade in over $85 \%$ of the cases. 
Table 3: Causes of stillbirths and neonatal deaths weighing $\geq 1500$ grams and maternal medical conditions

\begin{tabular}{|c|c|c|}
\hline Perinatal deaths & Number & $\%$ \\
\hline \multicolumn{3}{|l|}{ Still birth } \\
\hline Macerated & 18 & 13.5 \\
\hline Fresh & 78 & 58.7 \\
\hline Admitted with foetal heart beat & 32 & 24.1 \\
\hline Admitted without heart beat & 46 & 34.6 \\
\hline Early neonatal death & 37 & 27.8 \\
\hline Total & 133 & 100 \\
\hline \multicolumn{3}{|c|}{ Intrapartum causes of fresh stillbirth } \\
\hline Birth asphyxia & 37 & 47.4 \\
\hline Abruption Placenta & 18 & 23.2 \\
\hline Ruptured uterus & 5 & 6.4 \\
\hline Haemorrhage other than abruption & 3 & 3.8 \\
\hline Congenital anomaly & 3 & 3.8 \\
\hline Cord prolapsed & 2 & 2.6 \\
\hline Unknown & 10 & 12.8 \\
\hline Total & 78 & 100 \\
\hline \multicolumn{3}{|l|}{ Causes of early neonatal death } \\
\hline Birth asphyxia & 19 & 51.3 \\
\hline RDS & 8 & 21.6 \\
\hline Meconium aspiration & 3 & 8.1 \\
\hline Neonatal jaundice & 3 & 8.1 \\
\hline Head injury during delivery & 3 & 8.1 \\
\hline Aspiration pneumonitis & I & 2.7 \\
\hline Total & 37 & 100 \\
\hline \multicolumn{3}{|l|}{ Maternal medical condition } \\
\hline Eclampsia & 33 & 24.8 \\
\hline Anaemia & 19 & 14.3 \\
\hline Preeclampsia & 11 & 8.3 \\
\hline Severe anaemia & 6 & 4.5 \\
\hline HIVIAIDS & 5 & 3.8 \\
\hline Severe malaria & 2 & 1.5 \\
\hline Diabetes mellitus & I & 0.8 \\
\hline Others & 2 & 1.8 \\
\hline None & 54 & 41.3 \\
\hline Total & 133 & 100 \\
\hline
\end{tabular}

Given that $51.9 \%$ of the dead babies had heart beats on arrival and that suboptimal care was assessed as the likely reason for deaths in $43-53 \%$ by the auditors, it can be estimated that one out of four deaths could be prevented by improved standard of obstetric care.

The avoidable factors identified by the auditors are presented in Table 4 . The deficiencies in clinical care were dominating, mainly the professional performance but also the organisation. Rather few cases of patient related (community) delay were identified.
The two most important areas for improvement of obstetric care were monitoring during labour of the foetal condition and delay of clinical decisions as well as delay of implementation of decided actions. Lack of 24 hour comprehensive emergency obstetric care, and problems with transport from the municipal hospitals contributed to delays in referrals. Furthermore, ongoing hospital renovation at MNH caused severe shortage of theatre space; only one theatre for surgery in obstetrics and gynaecology was available during the study period.

Table 5 summarizes the level of agreement among auditors. On evaluation of the quality of care, there was a better agreement between the external auditors $(\kappa=0.25)$ as compared to the external auditors versus the internal auditor $(\kappa=0.08-0.19)$. However, there was a better agreement between external auditor no 2 and the internal auditor with respect to final grading and contribution of suboptimal factors to adverse outcome $\kappa=0.31-0.37$ and $0.30-0.35)$. There was a better agreement between the two external auditors in the questions about the adequacy of the information to assign the final grade and case preventability ( $\kappa=0.30$ and $\kappa=0.25$ respectively) as compared to both external and internal auditor.

\section{Monitoring during labour}

Poor foetal heart rate monitoring during labour was indirectly associated with over $40 \%$ of perinatal deaths. Among auditors, poor foetal monitoring had 57 (43\%), $55(41 \%)$ and $62(47 \%)$ agreement. Poor documentation also was a major problem as has been shown by the exclusion of many cases lacking information. The majority of patients with induction of labour were induced with vaginal misoprostol. The auditors found that the dose used was too high (50 microgram) and there was no clinical assessment of the foetus before induction to determine if the foetus would not be able to endure the induced labour due to severe dysfunction of the placental-foetal unit. This would have increased the risk of asphyxia caused by hyper-stimulation. It was also noted that patients with induced labour were poorly monitored. Partogram were not properly filled and foetal heart rate monitoring was inadequate.

\section{Delayed management}

A proportion of three quarters $(n=23)$ of patients referred to $\mathrm{MNH}$ took a very long time to reach $\mathrm{MNH}$ due to transport problems. In order to save fuel ambulances wait until there are more than two patients, furthermore, in municipal hospitals the same ambulance ferries patients from other wards as well, so there was a tendency of waiting irrespective of the degree of emergency. Some of the cases referred for CS could have been delivered at the referring hospital, which might have prevented foetal death. 
Table 4: Suboptimal care and its contribution to adverse perinatal outcome, level of delay and final grading by auditors

\begin{tabular}{|c|c|c|c|c|c|c|}
\hline & \multicolumn{2}{|c|}{ External I } & \multicolumn{2}{|c|}{ External 2} & \multicolumn{2}{|c|}{ Internal } \\
\hline & No & $\%$ & No & $\%$ & No & $\%$ \\
\hline \multicolumn{7}{|l|}{ Suboptimal factor: } \\
\hline Yes & 105 & 78.9 & 115 & 86.4 & 107 & 80.5 \\
\hline No & 19 & 14.3 & 15 & 11.3 & 16 & 12.0 \\
\hline No opinion & 9 & 6.8 & 3 & 2.3 & 10 & 7.5 \\
\hline \multicolumn{7}{|c|}{ Contribution of suboptimal factor to adverse outcome: } \\
\hline Unlikely & 9 & 6.8 & 25 & 18.8 & 23 & 17.3 \\
\hline Possibly & 30 & 22.5 & 35 & 26.3 & 49 & 36.8 \\
\hline Likely & 71 & 53.4 & 58 & 43.6 & 58 & 43.6 \\
\hline No opinion & 23 & 17.3 & 15 & 11.3 & 3 & 2.3 \\
\hline \multicolumn{7}{|l|}{ Level of delay: } \\
\hline Community/family level & 1 & 0.8 & 10 & 7.5 & 9 & 6.8 \\
\hline Infrastructure & 4 & 3.0 & 2 & 1.5 & 8 & 6.0 \\
\hline Care & 86 & 64.6 & 80 & 60.1 & 62 & 46.6 \\
\hline Infrastructure and care & 21 & 15.8 & 22 & 16.6 & 35 & 26.3 \\
\hline No comments & 21 & 15.8 & 19 & 14.3 & 19 & 14.3 \\
\hline \multicolumn{7}{|l|}{ Final grade: } \\
\hline 0 & 19 & 14.3 & 15 & 11.3 & 22 & 16.5 \\
\hline I & 11 & 8.3 & 19 & 14.3 & $\mathrm{II}$ & 8.3 \\
\hline ॥ & 54 & 40.6 & 42 & 31.6 & 65 & 48.9 \\
\hline III & 49 & 36.8 & 57 & 42.9 & 35 & 26.3 \\
\hline \multicolumn{7}{|c|}{ Was the information available sufficient to assign a final grade? } \\
\hline Yes & 116 & 87.2 & 120 & 90.2 & 114 & 85.7 \\
\hline No & 15 & 11.3 & 12 & 9.0 & 18 & 13.5 \\
\hline No opinion & 2 & 1.5 & 1 & 0.8 & 1 & 0.8 \\
\hline \multicolumn{7}{|c|}{ Do you consider likely that this death was preventable? } \\
\hline Yes & 101 & 75.9 & 69 & 51.6 & 105 & 78.9 \\
\hline No & 24 & 18.0 & 58 & 43.6 & 24 & 18.0 \\
\hline No opinion & 8 & 6.0 & 6 & 4.5 & 4 & 3.0 \\
\hline \multicolumn{7}{|l|}{ Avoidable factors detected by auditors: } \\
\hline Inadequate foetal heart monitoring during labour & 57 & 42.9 & 55 & 41.4 & 62 & 46.6 \\
\hline Delayed operation & 18 & 13.5 & 22 & 16.5 & 15 & 11.3 \\
\hline Delayed referral & 20 & 15.0 & 18 & 13.3 & 31 & 23.3 \\
\hline Failure to diagnose & 4 & 3.0 & 10 & 7.5 & 7 & 5.3 \\
\hline Traumatic assisted delivery & 2 & 1.5 & 1 & 0.8 & 1 & 0.8 \\
\hline Failure to resuscitate a newborn & 0 & 0 & 0 & 0 & 3 & 2.3 \\
\hline Inadequate antenatal care & 3 & 2.3 & 2 & 1.5 & 3 & 2.3 \\
\hline Others & 10 & 7.5 & 17 & 12.8 & 4 & 2.8 \\
\hline None & 19 & 14.3 & 8 & 6.0 & 7 & 5.3 \\
\hline Total & 133 & 100 & 133 & 100 & 133 & 100 \\
\hline
\end{tabular}

Table 5: Level of agreement among auditors

\begin{tabular}{lccc}
\hline Item & External I vs. External 2 & External I vs. Internal & External 2 vs. Internal \\
\hline Suboptimal factor (Yes, No) & 0.25 & 0.078 & 0.19 \\
Contribution of suboptimal factor to adverse outcome & 0.30 & 0.29 & 0.35 \\
Final grading (0, I, II, III) & 0.31 & 0.31 & 0.37 \\
Was the information available sufficient to assign a final grade & 0.30 & 0.05 & 0.05 \\
Do you consider likely that this death was preventable & 0.27 & 0.26 & 0.34
\end{tabular}


At $\mathrm{MNH}$, after making a decision to deliver by CS, there were very long intervals between decision and actual procedure. Some patients were delivered vaginally while waiting for CS, and instrumental vaginal delivery could have improved the outcome of these babies. The auditors suggested more frequent use of vacuum extraction (Ventouse) delivery. This procedure might have had decreased the adverse perinatal outcome and the events of vaginal delivery in the theatre for patients scheduled for emergency caesarean section (CS). In case of foetal distress, prompt abdominal delivery and transfer of the baby to the neonatal unit could have improved the outcome for many babies. Prolonged labour diagnosed at $\mathrm{MNH}$ as well as at the referring hospitals contributed probably substantially to intrapartum and early neonatal deaths.

Initiation of care at MNH was not immediate after the patient has been admitted. Even patients with seizures were not assessed by doctors within two hours after admission. All auditors concurred that delay in care was a major adverse factor for perinatal outcome.

Other comments by the auditors were related to failure to diagnose as well as to consult with more experienced doctors and this resulted in wrong management. There was often lack of communication between senior and junior doctors.

\section{Discussion}

Previous studies conducted in Tanzania and elsewhere put more emphasis on risk factors and adverse perinatal outcome instead of evaluating what is really happening during labour [14]. The current study is the first attempt at $\mathrm{MNH}$ to qualitatively identify potentially avoidable causes of perinatal deaths and suggest course of action to reduce perinatal mortality.

Previous studies have indicated that the majority of perinatal mortality is actually preventable without extra resource inputs. Perinatal audit in the South Western highlands of Tanzania by van Roosmalen [15] resulted in a reduction of perinatal mortality rate from 71 to 39/1000 following the introduction of a new obstetric policy that emphasized the prevention of prolonged labour, the early detection of foetal distress, and better recognition of women with high risk pregnancies.

According to this audit intrapartum deaths and neonatal deaths could be prevented by up to $64 \%$ according to external auditors and by $79 \%$ by internal auditor. These figures are much higher as compared to a similar study in Sudan [16]. It may be questioned at this point whether this type of qualitative audit improve care on itself. To achieve this prolonged follow-up studies are necessary to measure the impact like what has been done for ten years in Mozambique [17]. This needs resources and may necessitate relinquishing from other quality improvement activities and concentrate on measuring the effect of audit. However, it is ethical and very important to have continuous quality improvements. This study is the beginning of a continuous process to improve care in a hospital where audit was non-existent. This audit focused on adverse perinatal outcome and carries a risk encouraging interventions such as operative deliveries. Therefore, it is imperative that it should be complemented by other quality improvement activities rather than standing alone. However, this kind of audit process provides a good start for quality improvement in developing countries like Tanzania. It is obvious that it is not possible in a busy labour ward like MNH to prepare case notes of all cases for audit, but a good way is to give the task to one of the junior doctors to prepare case stories for assessment e.g. 2-3 cases a week that can be presented and assessed at the weekly meeting. One can alternate between types of cases as the senior obstetrician decides. In general practice it is not the main aim to get statistics of the sort we have in a paper, so there is no need to assess all cases as long as all types of complications are represented.

An audit has a great strength in quality assessment because of the continuous and systematic assessment by peer groups. It enables identification of unique aspects by comparison with established guidelines. Audit can bring change by continuous feedback to stake holders for the purpose of intervention. There are however, diverging opinions about the utility of perinatal audit, some report an effect in decreasing risk of perinatal mortality by improving the practice of health care providers while others claim that benefits have never been adequately evaluated [18]. Audit in poor resource countries like Tanzania is always compromised by the poor quality of information recorded in women's case notes, and by conflict between ideal care and available resources. In this study the poor quality of the patient records including lack of partogram has been identified as a problem. The improvement of recording practices by doctors and nurses is therefore recommended.

The high level of suboptimal care and concurrent avoidable perinatal death noted here is a finding similar to previous studies in Africa [19] but much higher than that found in European studies [20,21]. The current study used the term suboptimal care when there was a departure from acceptable evidence based standards. However, suboptimal care does not necessarily lead to a perinatal death, because it can be identified both in cases with good and adverse outcome. In contrast, avoidable factors always refer to their relationship with adverse outcome. This study graded levels of suboptimal care as an unlikely, possibly or a likely cause of the adverse perinatal outcome. 
About half of the audited deaths were likely to have been due to suboptimal care, and this indicates that changes in the daily routines can bring significant reduction in perinatal mortality in this urban tertiary centre.

The methodology used had the advantage that the auditors included both a local obstetrician and two external auditors. One of the external auditors had vast experience of obstetric practice in Africa and the other obstetrician had not practiced in Africa. We consider that this combination of auditors added strength to our study and might have minimized bias in the total assessment. The internal auditor was an obstetrician working in the same hospital which might have made her less critical, but she also had the advantage of knowledge of the working environment. It was more common that the external auditors considered that clinical action was delayed than the internal auditor; still, there was overwhelming agreement among auditors that about $80 \%$ of the perinatal deaths assessed were characterized by suboptimal care. The evaluation of the cases and grading depended greatly on the individual auditors experience as reflected by fair to poor agreement among auditors. The external auditors were more critical than the internal auditor. It would be questioned as to whether external auditors should always be invited in this kind of audit, no, however, for this particular study it was necessary because it was the introduction of an audit in this hospital with very high perinatal mortality, therefore, it was as well a capacity building process.

Although the level of delay ranged from the patient herself to the health care in the institution, this audit was primarily concerned with the type of service the patient received after arrival in the health institution. This is also the most useful part of an audit, because it is within the institution that change can be instituted immediately. Lack of proper foetal monitoring during labour at $\mathrm{MNH}$ has nothing to do with problems in the community or in the referring hospitals.

The main limitations of this study are the inability to include events during the antenatal period and many of the perinatal death due to inadequate documentation. The finding of almost equal numbers of fresh and macerated stillbirths suggests that many problems were linked to insufficient care during the antenatal period. Previous studies done in Tanzania $[22,23]$ and elsewhere [24] revealed that poor antenatal care and infections are associated with antepartum stillbirths. In our material one third of the perinatal deaths were related to eclampsia and preeclampsia that were first diagnosed at admission, a clear indication of inadequate antenatal care. A number of recent studies have questioned the effectiveness of some components of antenatal care in reducing the risk of poor pregnancy outcomes. In particular, questions have been raised on the effectiveness of maternal weight and blood pressure measurements, and a too high frequency of visits [25]. It is, therefore, necessary that the limited resources available target those components of antenatal care proven to be the most cost-effective. When hypertension is diagnosed during ANC, it should be evaluated and followed up; otherwise complications like eclampsia will not be prevented.

Poor documentation is a major bottleneck in most developing countries; in this study many case notes lacked important information like Apgar score, type of stillbirth (Macerated still birth (MSB) or Fresh still birth (FSB)), sex of the baby etc. This necessitated exclusion of these cases from the study; however, since there were a big number of cases for analysis we consider that excluded cases are not a major source of bias.

$\mathrm{MNH}$ is the only public referral tertiary hospital in Dar es Salaam, the three municipal hospitals do not have an effective round the clock comprehensive emergency obstetric care, and therefore, most cases which needed emergency operative delivery and blood transfusion were referred to $\mathrm{MNH}$ where there was a long queue for operation. Delayed operative delivery was indicated as one of the avoidable factors. The almost non-use of instrumental vaginal delivery did also contribute to delays during the second stage of labour resulting in birth asphyxia and perinatal death. During the study period there was major renovation of the obstetric theatre and only one operating room was available. This might also have contributed to the delays in care.

In the prevention of perinatal deaths significant areas remain for health care improvements and this audit highlights the importance of care during labour and delivery. According to the findings, about one in 4 perinatal deaths in this tertiary centre could be attributed to avoidable factors linked to obstetric care. The most important policies to implement in the future are adequate foetal and maternal monitoring during labour, shortening the interval between decision and caesarean section, performing instrumental vaginal delivery when indicated, reducing prolonged labour at $\mathrm{MNH}$ as well as in referring hospitals and promote prompt hospital transfer after decision to refer and helping the baby to breath by immediate resuscitation at delivery. Another example of suboptimal practise is that eclampsia patients were induced with high dose of misoprostol without pre-induction assessment whether the foetus was compromised in relation to growth and/or placental insufficiency, and together with lack of proper foetal heart rate monitoring this may have resulted in asphyxia and foetal demise.

The population of Dar es Salaam has kept on growing whereas the public health facilities have remained at the same level as previously. This has resulted in congestion at 
this large referral centre and too many patients who do not need specialized care. To improve the services at $\mathrm{MNH}$ establishing new public maternities in the city is necessary. The hospital will then be able to care better for patients who really need specialized care.

\section{Intervention}

For intervention purposes the following steps have been taken to improve the perinatal outcome. These steps will be followed by data collection to assess the impact.

- Continued medical education by re-training the midwife and doctors on the use of parthogram and interpretation of abnormal labour and helping asphyxiated babies to breath (Two trainings have been conducted with 60 participants each). This will improve monitoring during labour and resuscitation of asphyxiated newborn.

- Management protocols for eclampsia and other obstetrics emergences have been prepared and displayed in the wards notice boards.

- New sets (5 sets) of vacuum (ventouse) and Doppler machines have been purchased for assisted deliveries and assessment of foetal heart beats.

- Nurses/midwives have started routine continued medical education every morning once a week.

- A decision operation interval is checked by record tracing of the patient from the labour ward to theatre. (A log book has been opened for this purpose to identify areas of delay).

- The administration has been in contact with the municipal hospitals to streamline referrals so that there are no delays.

- An audit committee has been set up. Regular perinatal audits have been introduced every last Tuesday of the month there is a departmental perinatal audit meeting. Daily assessments of all perinatal deaths by the team on call. Weekly assessments by the audit team

- Documentation has been stressed and a new slogan not documented not done has been put in place.

- An obstetrician on call has been station in the labour ward to assist on evaluation and decision making.

\section{Conclusion}

Regular perinatal audits to identify avoidable causes of perinatal deaths as well as feed back to the health care pro- viders has the potential to reduce perinatal mortality in this large African urban hospital by $25 \%$. However, this should involve the hospital management and the municipal hospitals in the city that are the referring hospitals. Priority interventions are proper monitoring of patients during labour, assessment of the foetus before decision of route of delivery, early referrals and prompt instrumental and surgical intervention. Training on monitoring labour and newborn resuscitation to newly employed midwives and doctors shall be regularly conducted. Development of management guidelines will be done before reassessing the level of improvement.

\section{Competing interests}

The authors declare that they have no competing interests.

\section{Authors' contributions}

HLK, participated in design of the study carried out the data collection and the analyses and drafted the first and last manuscript. IM participated in design of the study, was one of the auditors and reviewed the manuscript. JVR was one of the auditors and reviewed the manuscript. ANT was one of the auditors and reviewed the manuscript. SNM participated in designing the study and supervised the data collection LN participated in the analysis of data, and development of the manuscript. GL supervised and participated in all steps of the study.

\section{Acknowledgements}

We wish to acknowledge SIDA/SAREC and the global health for their financial support, which enabled this study to be done, we also thank the Muhimbili National Hospital administration for allowing us to conduct the study in the hospital. This work was partly supported by the Umeå Centre for Global Health Research, with support from FAS, the Swedish Council for Working Life and Social Research (grant no. 2006-15/2).

\section{References}

I. National Bureau of Statistics and Calverton: Tanzania demographic and health survey (TDHS) 2004-05. Dar es Salaam, Tanzania, 2005. MD, USA: ORC Macro.

2. WHO, The World Health Report 2005: Make every mother and child count. WHO, Geneva, Switzerland; 2005.

3. Lawn J, Shibuya K, Stein C: No cry at birth: global estimates of intrapartum stillbirths and intrapartum-related neonatal deaths. Bull WHO 2005, 83(6):409-4I7.

4. Kramer MS: The epidemiology of adverse pregnancy outcomes. An overview. J Nutr 2003, I 33(5 Suppl 2): $1592 S-1596 S$.

5. Hinderaker SG, Olsen BE, Bergsjo PB, Gasheka P, Lie RT, Haven J Kvale G: Avoidable stillbirths and neonatal deaths in rural Tanzania. Br J Obstet Gynaecol 2003, I I 0:616-623.

6. Kidanto H, Massawe SN, Nystrom L, Lindmark G: Analysis of perinatal mortality at a teaching hospital in Dar es Salaam, Tanzania, 1999-2003. Afr J Repr Health 2006, 10:73-80.

7. Wilkinson D: Reducing perinatal mortality in developing countries. Health Policy Plan 2002, I 2:16|-165.

8. Pattinson RC: Challenges in saving babies, avoidable factors, missed opportunities and substandard care in perinatal deaths in South Africa. South Afr Med J 2003, 93:450-455.

9. Mancey-Jones M, Brugha R: Using perinatal audit to promote change: a review. Health Policy Plan 1997, I2:183-192.

10. Drife JO: Perinatal audit in low- and high-income countries. Semin Fetal Neonat Med 2006, I I:29-36. 
II. Richardus JH, Graafmans WC, Verloove-Vanhorick SP, Mackenbach JP: The perinatal mortality rate as an indicator of quality of care in international comparisons. Med Care 1998, 36:54-66.

12. Hanne BC, Langhoff-Roos J, Larsen S, Lindberg B, Wennergren M: The Nordic/Baltic perinatal death classification. Acta Obstet Gynaecol Scand 1997, I 64(Suppl):40-42.

13. Landis J, Koch G: The measurement of observer's agreement for categorical data. Biometrics 1977, 33: I59-874.

14. De Muylder X: Perinatal mortality audit in a Zimbabwean district. Pediatr Perinatal Epidemiol 1989, 3:284-293.

15. Van Roosmalen J: Perinatal mortality in rural Tanzania. $\mathrm{Br} J$ Obstet Gynaecol 1989, 96:827-834.

16. El Amin S, Langhoff-Roos J, Bakr Ali Abu, Abdu Latif A, Salah A, Lindmark G: Introducing qualitative perinatal audit in a tertiary hospital in Sudan. Health Policy Plan 2002, 1 7:296-303.

17. Bughalo A, Bergstrom S: Value of perinatal audit in obstetric care in the developing country: $A$ ten year experience of the Maputo model. Gynecol Obstet Invest 1993, 36:239-243.

18. Feresu SA, Harlow SD, Welch K, Gillespie BW: Incidence of stillbirth and perinatal mortality and their associated factors among women delivering at Harare Maternity Hospital, Zimbabwe: a cross-sectional retrospective analysis. BMC Pregnancy Childbirth 2005, 5:9.

19. Kuti O, Orjie O, Ogunlola O: Analysis of perinatal mortality in a Nigerian teaching hospital. J Obstet Gynaecol 2003, 23:5 I 2-5 I4

20. Saastad E: Suboptimal care in stillbirths, a retrospective audit. Evaluation of the prenatal care and possibilities for quality improvements with special focus on non-western women. In Thesis University of Oslo, Norway; 2006.

21. Richardus JH, Graafmans WC, Bergsjo P, Lloyd DJ, Bakketeig LS, Bannon EM: Sub optimal care and perinatal mortality in ten European regions: methodology and evaluation of an international audit. J Mater-Fetal Neonat Med 2003, I 4:267-276.

22. Watson-Jones D, Weiss H, Changalucha J, Todd J, Gumodoka B, Bulmer J, Balira R, Ross D, Mugeye K, Mabey D, Hayes R: Adverse birth outcomes in United Republic of Tanzania, impact and prevention of maternal risk factors. Bull WHO 2007, 85:9-18.

23. Mbaruku G, van Roosemalen J, Kimondo I, Bilango , Bergstrom S: Perinatal audit using a 3-delay model in western Tanzania. Int J Obstet Gynecol 2009, I 06:85-88.

24. Lawn JE, Yakoob MY, Haws R, Soomro T, Darmstatadt GI, Bhutta Z: 3.2 million stillbirth: Epidemiology and overview of the evidence review. BMC Pregnancy Childbirth 2009, 9(SuppI I):S2.

25. Munjanja SP, Lindmark G, Nystrom L: Randomized controlled trial of a reduced-visits programme of antenatal care in Harare, Zimbabwe. Lancet 1996, 348:364-369.

\section{Pre-publication history}

The pre-publication history for this paper can be accessed here:

http://www.biomedcentral.com/1471-2393/9/45/prepub
Publish with Biomed Central and every scientist can read your work free of charge

"BioMed Central will be the most significant development for disseminating the results of biomedical research in our lifetime. "

Sir Paul Nurse, Cancer Research UK

Your research papers will be:

- available free of charge to the entire biomedical community

- peer reviewed and published immediately upon acceptance

- cited in PubMed and archived on PubMed Central

- yours - you keep the copyright
BioMedcentral 